Discrete variational quantum reactive scattering method with optimal distorted waves. II. Application to the reaction $\mathrm{H}_{+} \mathrm{O}_{2} \rightarrow \mathrm{OH}+\mathrm{O}$

Gerrit C. Groenenboom

Citation: The Journal of Chemical Physics 108, 5677 (1998); doi: 10.1063/1.475977

View online: http://dx.doi.org/10.1063/1.475977

View Table of Contents: http://aip.scitation.org/toc/jcp/108/14

Published by the American Institute of Physics

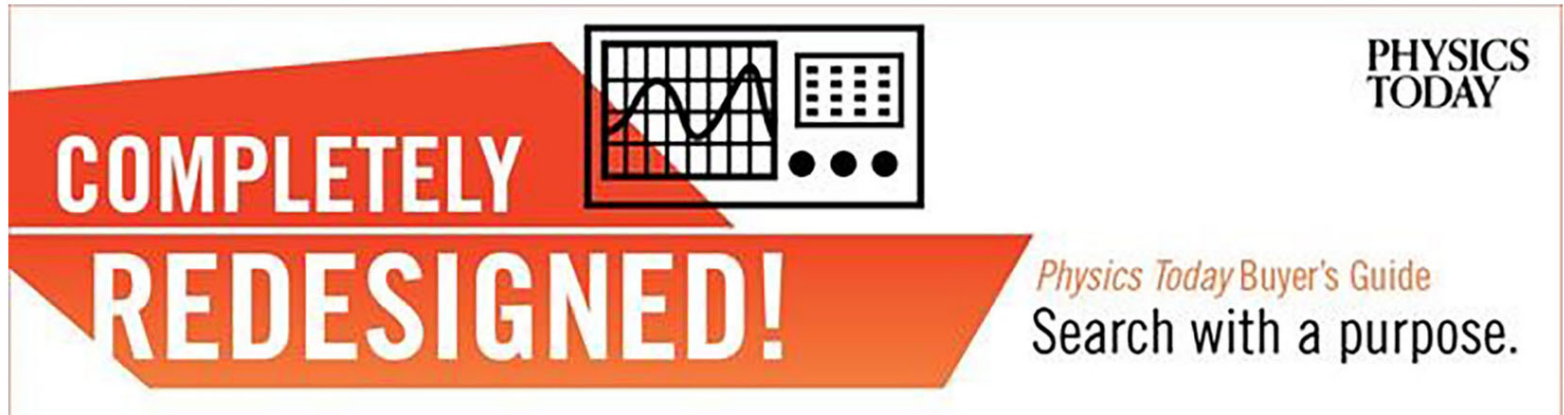




\title{
Discrete variational quantum reactive scattering method with optimal distorted waves. II. Application to the reaction $\mathrm{H}+\mathrm{O}_{2} \rightarrow \mathrm{OH}+\mathrm{O}$
}

\author{
Gerrit C. Groenenboom \\ Institute of Theoretical Chemistry, NSR Center, University of Nijmegen, Toernooiveld, 6525 ED Nijmegen, \\ The Netherlands
}

(Received 10 October 1997; accepted 8 January 1998)

\begin{abstract}
The discrete Kohn variational reactive scattering method presented in the preceding paper is applied to the reaction $\mathrm{H}+\mathrm{O}_{2} \rightarrow \mathrm{OH}+\mathrm{O}$. The essential features of the method are the use of an interaction region grid, fully coupled open and closed distorted waves (DWs) and the use of a singular value decomposition technique to construct the optimal boundary conditions for the open DWs. A convergence test is presented at a total energy of $0.817 \mathrm{eV}$ above the bottom of the $\mathrm{H}+\mathrm{O}_{2}$ well. It is shown that very well converged results may be obtained in a calculation with a relatively small interaction region grid, when at least a few asymptotically closed DWs are included in the trial wave function. Furthermore, the number of open distorted waves (DWs) may be considerably smaller than the number of open channels. Six additional points are computed in an energy range of $1.2 \mathrm{meV}$, scanning through a narrow resonance around $0.817 \mathrm{eV}$. The results are in very good agreement with the hyperspherical coordinate propagation calculations by R. T Pack, E. A. Butcher, and G. A. Parker [J. Chem. Phys. 102, 5998 (1995)]. (C) 1998 American Institute of Physics.
\end{abstract}

[S0021-9606(98)01614-6]

\section{INTRODUCTION}

The reaction,

$\mathrm{H}+\mathrm{O}_{2}\left({ }^{3} \Sigma_{g}^{-}, v, j\right) \rightarrow \mathrm{OH}\left({ }^{2} \Pi, v^{\prime}, j^{\prime}\right)+\mathrm{O}\left({ }^{3} P\right)$,

which is endothermic by about $0.71 \mathrm{eV}$, is generally considered to be difficult for dynamical calculations, because of the deep well of $\sim 2.38 \mathrm{eV}$ corresponding to the stable $\mathrm{HO}_{2}$ radical. Furthermore, the potential has a long ranging dipolequadrupole $\left(R^{-4}\right)$ behavior in the $\mathrm{O}+\mathrm{OH}$ arrangement. In the last three years several non-variational-yet rigorousthree-dimensional $(J=0)$ quantum dynamics calculations have been reported for this reaction. These include the cumulative reaction probability calculation by Leforestier and Miller, ${ }^{1}$ the initial state selected, time dependent calculations by Zhang and Zhang ${ }^{2}$ and full state-to-state, adiabatically adjusting principal axes hyperspherical $(\mathrm{APH})$ coordinates coupled channel calculations by Pack, Butcher, and Parker (PBP). ${ }^{3}$ In the present paper we report very well converged state-to-state results for the $\mathrm{H}+\mathrm{O}_{2}$ reaction, obtained by the variational method described in paper $\mathrm{I}^{4}{ }^{4}$ This method combines a grid representation for the wave function in the interaction region with a set of fully coupled distorted waves (DWs) to account for the elastic and inelastic effects of the long range interactions. An iterative method is used to solve the linear equations that form the most time-consuming step in the variational formalism. Here, we investigate the convergence of the calculation with respect to both the grid- and the DW-parameters. In particular, we demonstrate that the singular value associated with each (open) DW, as defined in paper I, is a very good a priori measure of the contribution of that DW to the full $S$-matrix. The minimization of the number of DWs is very useful because the total computation time scales linearly with the number of DWs when an itera- tive method is used to solve the linear equations. Furthermore, we show that the use of closed DWs-also defined in paper I-is essential for obtaining highly converged results with a minimum grid size. Since in this formalism the DWs are defined to be well converged solutions of the Schrödinger equation in the external regions (i.e., the regions outside the interaction region grid), the minimum extent of the interaction region grid is determined by the condition that the DWs of different arrangements do not overlap.

We use the DMBE IV potential energy surface of Pastrana et $a l .{ }^{5}$ and we make the convergence tests at a total energy of $E=0.817 \mathrm{eV}$ above the $\mathrm{H}+\mathrm{O}_{2}$ well (thus, $E-V_{\min }=3.195355 \mathrm{eV}$, where $V_{\min }$ is the minimum of the potential). We compare our results with the results of the APH calculation of PBP, who also present a convergence test at this energy. Furthermore, we compute the $S$-matrices at six additional energies in the interval between 0.8168 and $0.8180 \mathrm{eV}$ where PBP found a very narrow resonance; the cumulative reaction probability $\left[N^{0}(E)\right]$, which is a rather averaged quantity, varies by almost a factor of three in this interval. The results are in good agreement with PBP, even at this high energy resolution.

Note that the main purpose of the present study is to test the new dynamical method. A physically realistic description of the details of the reaction should include the geometric phase effect, as was recently shown by Kendrick and Pack in a study employing a new potential energy surface. ${ }^{6}$ In addition, the open shell character of the $\mathrm{OH}$ and conical intersections ${ }^{7}$ can be expected to have important consequences. Also, the $J>0$ contributions should be taken into account.

In the next section we define the parameters used in the calculations. In Sec. III we present the results, the conver- 
TABLE I. The "large" and "small" sets of channel eigenfunctions used in the expansion of the DWs. There are $n_{\text {tot }}$ channels with energy less than $E_{\max }$ and the number of open channels is $n_{o}$. For all channels $j \leqslant j_{\max }$ and $v \leqslant v_{\max }$. The last two entries are the number of open DWs and the number of closed DWs used in the variational calculation.

\begin{tabular}{lcccccc}
\hline \hline & \multicolumn{2}{c}{ Arrangement 1} & & \multicolumn{2}{c}{ Arrangement 2} \\
\cline { 2 - 3 } \cline { 5 - 6 } \cline { 5 - 6 } & large & small & & large & small \\
\hline$E_{\max }-E(\mathrm{eV})$ & 1.60 & 1.48 & & 2.25 & 2.09 \\
$n_{\text {tot }}$ & 509 & 467 & & 141 & 127 \\
$n_{o}$ & 95 & 95 & & 2 & 2 \\
$j_{\max }$ & 109 & 105 & & 32 & 31 \\
$v_{\max }$ & 13 & 12 & & 5 & 5 \\
$\#$ open DWs & 55 & 50 & & 2 & 2 \\
$\#$ closed DWs & 6 & 4 & & 8 & 7 \\
\hline \hline
\end{tabular}

gence tests, the comparison with PBP and some timings. Section 4 contains the conclusion.

\section{PARAMETERS}

The boundary between the internal region (the grid) and the external region is defined by the values of the scattering coordinates $\left(R_{\alpha}=B_{\alpha}\right)$ of the dividing surfaces in each arrangement $(\alpha=1,2$, and 3 correspond to, respectively, $\mathrm{H}+\mathrm{O}_{2}, \mathrm{OH}+\mathrm{O}$, and $\left.\mathrm{O}+\mathrm{HO}\right)$. The minimum size of the interaction region is constrained by the condition that DWs of different arrangements do not overlap, which can be checked numerically before the solution of the linear equations. If symmetry is used in the calculation, one must be careful to also check the overlap between the two symmetry-related arrangements $\mathrm{OH}+\mathrm{O}$ and $\mathrm{O}+\mathrm{HO}$, even though the DWs are only computed for one of them. We found that if the interaction region is chosen too small, and hence the overlap between the DWs is not negligible, the computed $S$-matrix fails to be unitary, even if one attempts to converge the calculation with respect to all other coordinates. In the present calculations the size of the grid is given by $B_{1}=5$ bohr and $B_{2}=B_{3}=5.25$ bohr. Notice that the size of this grid is much smaller than the grids used in calculations with methods that do not employ DWs. For example, both the timeindependent calculation of Ref. 1 and the time-dependent calculation of Ref. 2 employ grids that extent outward in arrangement 1 at least twice as far.

The overlap between the DWs and the grid must be sufficiently large so that $\hat{H}-E$ applied to the regularized DWs is well represented on the grid. In our calculations we take the innermost boundary $\left(A_{\alpha}\right)$ such that $B_{\alpha}-A_{\alpha} \approx 3 \lambda_{\alpha}$, where $\lambda_{\alpha}$ is the de Broglie wavelength for the scattering coordinate, computed at the minimum of the potential, rather than from the asymptotic translational energy. Thus, we have $A_{1}=4.08$ bohr and $A_{2}=A_{3}=4.93 \mathrm{bohr}$. The cutoff-function used to regularize the DWs is defined in paper $\mathrm{I}$.

In the $\mathrm{H}+\mathrm{O}_{2}$ arrangement the DWs are propagated outward to $C_{1}=20 \mathrm{bohr}$, and in the $\mathrm{OH}+\mathrm{O}$ arrangement we propagate outward to $C_{2}=C_{3}=40 \mathrm{bohr}$, because of the long range dipole-quadrupole interaction.

In Table I we define the "large" and the "small" sets of channel eigenfunctions for each arrangement, that are used in
TABLE II. The grid parameters: the number of points $\left(n_{B}\right)$ per de Broglie wavelength (computed at the equilibrium geometry), the number of GaussLegendre points $n_{\theta}$ (divided by two because of symmetry), the cutoff potential $V_{\text {cut }}$ (relative to the minimum of the potential energy surface) and the grid spacings $\Delta_{R}$ and $\Delta_{r}$ computed from $n_{B}$. The number of grid points after the truncation of the grid using $V_{\text {cut }}$ is $n_{1}$. The actual number of grid points used $n_{\text {point }}$ is obtained by a further truncation of the grid using a technique described in Ref. 11.

\begin{tabular}{lccccccc}
\hline \hline Grid & $n_{B}$ & $n_{\theta}$ & $\begin{array}{c}V_{\text {cut }} \\
(\mathrm{eV})\end{array}$ & $\begin{array}{c}\Delta_{R} \\
(\mathrm{bohr})\end{array}$ & $\begin{array}{c}\Delta_{r} \\
(\mathrm{bohr})\end{array}$ & $n_{1}$ & $n_{\text {point }}$ \\
\hline large & 3.750 & $64 / 2$ & 12.50 & 0.0819 & 0.0286 & 220486 & 140407 \\
small & 3.375 & $58 / 2$ & 11.25 & 0.0910 & 0.0318 & 156836 & 103568 \\
\hline \hline
\end{tabular}

the convergence test below. The full set of eigenfunctions is used in the coupled channels expansion in the internal region $\left[A_{\alpha}, B_{\alpha}\right]$. In the external region $\left[B_{\alpha}, C_{\alpha}\right]$, which is divided in 16 logarithmically spaced intervals $\left[R_{i}, R_{i+1}\right]$, the number of channels used in each interval is scaled linearly with $R$ according to

$$
n_{i}=n_{o}+\operatorname{int}\left[n_{c}\left(R_{i}-C_{\alpha}\right) /\left(B_{\alpha}-C_{\alpha}\right)\right] \text {, }
$$

where $n_{o}$ is the number of open channels, $n_{c}$ the number of closed channels and "int" means rounded to the nearest integer. All other parameters for the construction of the DWs, such as the sector sizes (see Appendix B of paper I) and the quadratures to compute the W-matrices, are chosen such that they have a negligible contribution to the overall error.

The $(J=0)$ interaction region DVR, defined in Jacobi coordinates of arrangement $1(R, r, \theta)$, is similar to that described in a previous paper. ${ }^{8}$ Table II gives the parameters for the "large" and "small" grids which are used in the convergence tests below. For the scattering coordinate $(R)$ we use a $[0, \infty\rangle$ infinite order (or "wrapped" sinc-function) $\mathrm{DVR},{ }^{8-10}$ for the vibrational coordinate we use the $[-\infty, \infty]$ sinc-function DVR, and for the angular variable $(\theta)$ we use an odd symmetry Gauss-Legendre DVR. The direct product grid is truncated in two steps. First, we eliminate all points with an energy larger than $V_{\text {cut }}$. A relatively high cutoff energy is required in order to retain important points in certain repulsive parts of the potential. In the second step the number of grid points is reduced further by eliminating unnecessary points in the classically forbidden region, with a technique which will be described elsewhere. ${ }^{11}$

The high kinetic energy components of the angular DVR are eliminated with the projection technique described in Appendix A of Ref. 8. We apply the projection to each set of grid points with a given $\left(R_{i}, r_{j}\right)$ for which the angular kinetic energy

$$
T_{j}=\left(\frac{\hbar^{2}}{2 \mu R_{i}^{2}}+\frac{\hbar^{2}}{2 m r_{j}^{2}}\right) j_{\max }\left(j_{\max }+1\right)
$$

is larger than $20 \mathrm{eV}\left(j_{\max }=n_{\theta}-1\right)$. For such rows in the grid, points with $V>V_{\text {cut }}$ are set to $V=V_{\text {cut }}$, rather than being eliminated. ${ }^{12}$ This procedure reduces the spectral range of the Hamiltonian matrix and thus the number of iteration required to solve the linear systems. 

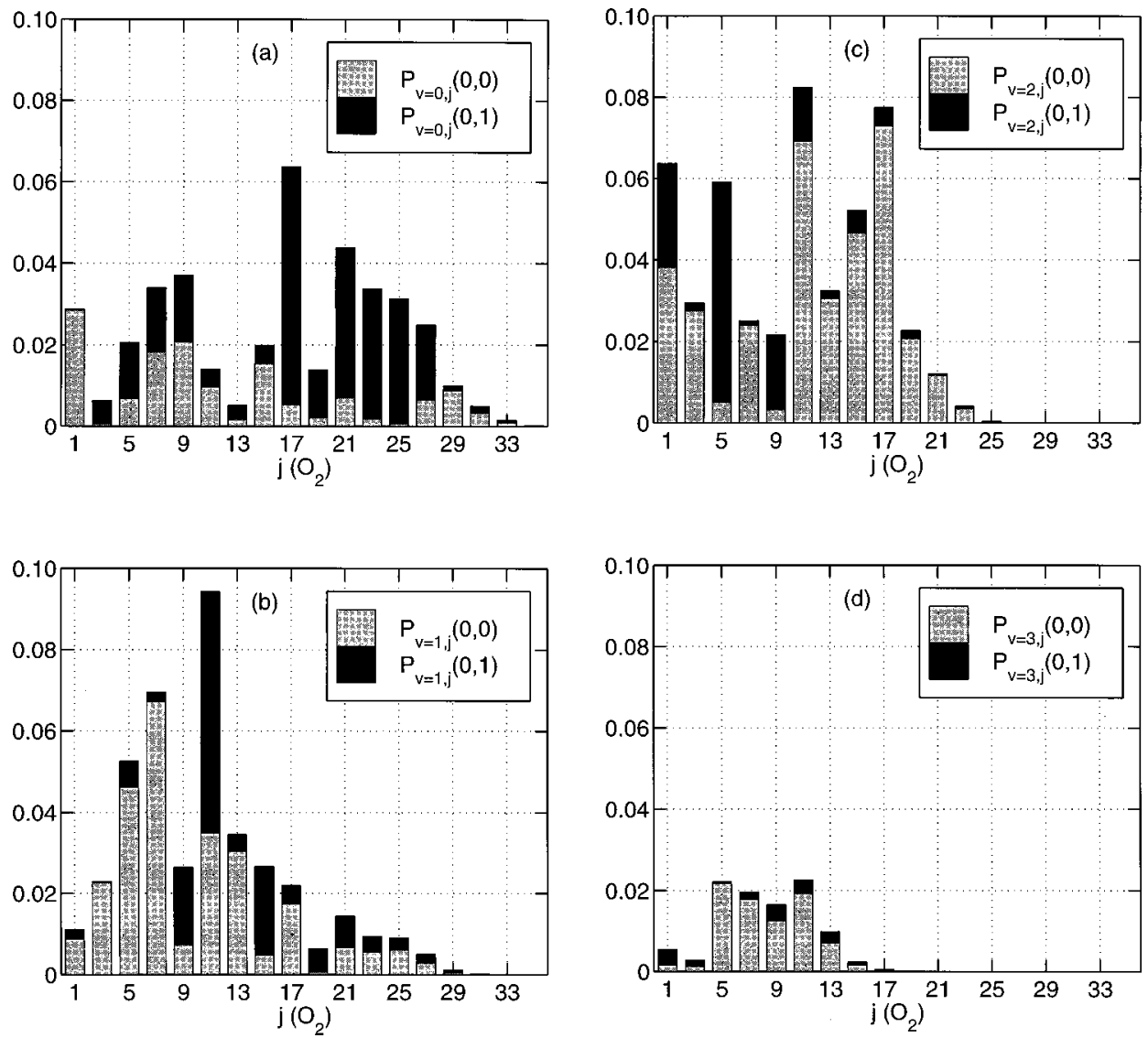

FIG. 1. The reaction probabilities for all $\mathrm{O}_{2}(v, j)$ initial states and all $\mathrm{OH}\left(v^{\prime}, j^{\prime}\right)$ final states.

\section{RESULTS}

In Fig. 1 we show the reaction probabilities $P_{v, j}\left(v^{\prime}, j^{\prime}\right)$ for all initial states of $\mathrm{O}_{2}(v, j)$ to both final states of $\mathrm{OH}$ $\left(v^{\prime}=0, j^{\prime}=0\right.$ or 1$)$ at a total energy of $E=0.817 \mathrm{eV}$, for a calculation using the best DWs and grid defined in Tables I and II. Although the main purpose of this paper is to demonstrate the method and to assess its convergence behavior we believe that it is interesting to present the simple analysis given in Table III. For each value of the $\mathrm{O}_{2}$ vibrational quantum number $(v)$ we determine the highest value of the rotational quantum number $(j)$ for which the total reaction probability is not negligible (i.e., larger than 0.001). Since for total $J=0$ the orbital angular momentum quantum number $l$ is equal to $j$, we may compute the corresponding classical impact parameter $(b)$ of this $\mathrm{O}_{2}(v, j)$ state via

$$
b \approx \hbar\left[\frac{l(l+1)}{2 \mu\left(E-\epsilon_{v, j}\right)}\right]^{1 / 2},
$$

TABLE III. The estimated largest impact parameter $(b)$ that contributes to the initial $\mathrm{O}_{2}$ vibrational state selected reaction probability $P_{v}$ (tot).

\begin{tabular}{cccccc}
\hline \hline$v$ & $l(=j)$ & $j_{\text {max open }}(v)$ & $E-\epsilon_{v, j}(\mathrm{eV})$ & $b(\mathrm{bohr})$ & $P_{v}($ tot $)$ \\
\hline 0 & 33 & 63 & 0.520 & 4.1 & 0.394 \\
1 & 29 & 53 & 0.373 & 4.2 & 0.406 \\
2 & 23 & 43 & 0.239 & 4.2 & 0.483 \\
3 & 15 & 27 & 0.0947 & 4.4 & 0.102 \\
\hline \hline
\end{tabular}

where $\mu$ is the reduced scattering mass for $\mathrm{H}+\mathrm{O}_{2}$ and $\epsilon_{v, j}$ is the asymptotic $\mathrm{O}_{2}$ channel energy. This analysis shows that at this total energy, a lower translational energy $\left(E-\epsilon_{v, j}\right)$ allows reactive trajectories with larger impact parameters. This behavior is consistent with the absence of a potential barrier in the entrance channels. Still, the vibrational state corresponding to the lowest translational energy gives the smallest reaction probability $P_{v}$ (tot), which suggests that the energy is not completely randomized on the time scale of the reaction.

\section{A. Convergence}

With the present method, as with the original $S$-matrix Kohn method, the unitarity of the computed $S$-matrix depends on the convergence of the calculation. Table IV shows that the deviation from unitarity, defined as the largest element of the matrix $\left|\boldsymbol{S}^{\dagger} \boldsymbol{S}-\mathbf{1}\right|$, is extremely small $\left(4 \times 10^{-9}\right)$ for the most extensive calculation. This, of course, does not

TABLE IV. Error estimates as defined in Sec. III A.

\begin{tabular}{llccccc}
\hline \hline \multirow{2}{*}{ Grid } & \multirow{2}{*}{ DW } & Unitarity & "Born”, & Arr. 1 & Arr. 2 & Reactive \\
\cline { 5 - 7 } & & & & & & \\
\cline { 5 - 7 } large & large & $4.0 \mathrm{e}-9$ & $3.1 \mathrm{e}-5$ & $0^{\mathrm{a}}$ & $0^{\mathrm{a}}$ & $0^{\mathrm{a}}$ \\
small & large & $1.2 \mathrm{e}-7$ & $2.3 \mathrm{e}-4$ & $4.4 \mathrm{e}-3$ & $4.7 \mathrm{e}-4$ & $1.3 \mathrm{e}-3$ \\
small & small & $1.6 \mathrm{e}-6$ & $4.5 \mathrm{e}-4$ & $4.4 \mathrm{e}-3$ & $4.8 \mathrm{e}-4$ & $1.4 \mathrm{e}-3$ \\
\hline \hline
\end{tabular}

${ }^{\mathrm{a} B y}$ definition. 

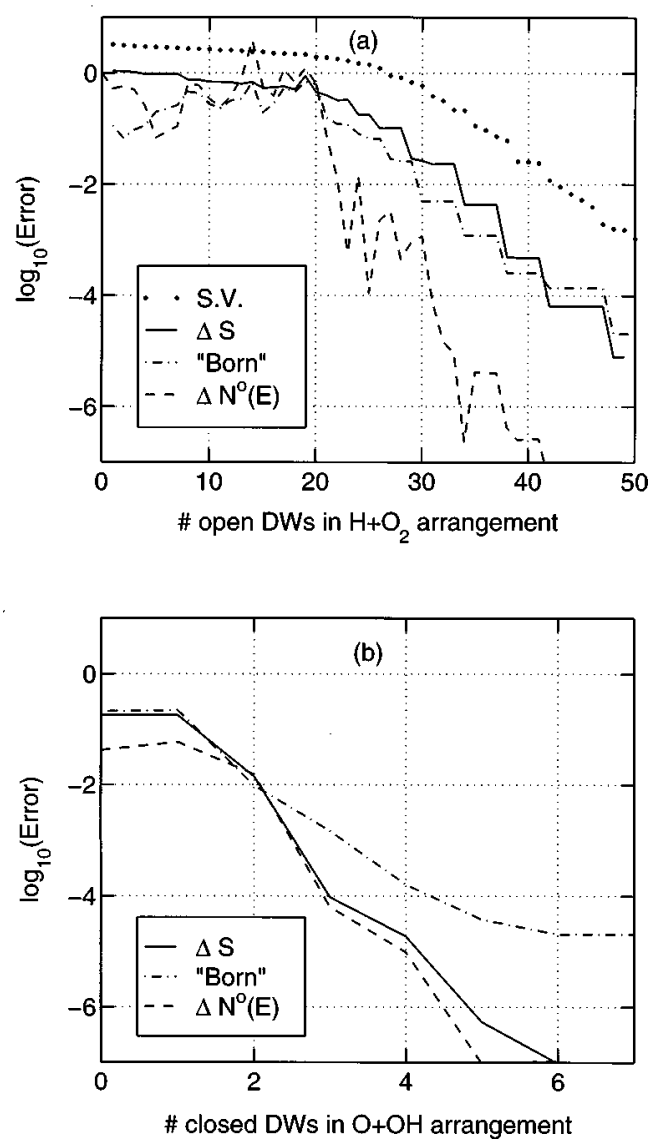

FIG. 2. The convergence as a function of (a) the number of open DWs in the $\mathrm{H}+\mathrm{O}_{2}$ arrangement and (b) the number of closed DWs in the $\mathrm{OH}+\mathrm{O}$ arrangements. The error estimates are defined in Sec. III A. S.V. is the singular value corresponding to each open DW, as defined in paper I.

guarantee the convergence of individual $S$-matrix elements, but at the very least it shows, as we discussed above, that the size of the interaction region grid is sufficiently large.

Another measure for the convergence of the calculation, also shown in Table IV, is the contribution to the $\boldsymbol{T}$-matrix from the second term in Eq. (23) of paper I (the "Born" term), which may easily be computed as the difference of the $\boldsymbol{T}$-matrix [Eq. (24)] and the trial $\widetilde{\boldsymbol{T}}$-matrix [Eq. (34)]. For a fully converged calculation the Born term must vanish. Comparing the $S$-matrices for calculations with different parameter sets is the best way to test the convergence. In Table IV we show the absolute value of the largest change in the $S$-matrix $(\Delta S)$, when comparing to the most expensive calculation. This error estimate is presented separately for the (in)elastic $\boldsymbol{S}$-matrix elements of arrangement 1 and 2, and for the reactive elements. Below, we shall see that in general the Born error estimate correlates reasonably well with $\Delta S$. This suggests that the largest deviation in any $\boldsymbol{S}$-matrix element in the most expensive calculation is in the order of $5 \times 10^{-4}$. The relative errors in the smaller $\boldsymbol{S}$-matrix elements may be larger than this, whereas more averaged quantities such as the cumulative reaction probability $\left[N^{0}(E)\right]$ and initial state selected total reaction probabilities may have a smaller relative error.

In Fig. 2(a) we show the convergence with respect to the number of open DWs in arrangement 1 . The solid line is
TABLE V. Convergence test and comparison with the APH calculation of PBP (Ref. 3) for the cumulative reaction probability $N^{0}(E)$, some initial state selected reaction probabilities $P_{v, j}($ total), and a single $\boldsymbol{P}$-matrix element $P_{v, j}\left(v^{\prime}, j^{\prime}\right)$.

\begin{tabular}{lcccccc}
\hline \hline Grid & \multirow{2}{*}{$\mathrm{DW}$} & $N^{0}(E)$ & $P_{0,1}($ tot $)$ & $P_{0,29}($ tot $)$ & $P_{3,1}$ (tot) & $P_{0,1}(0,0)$ \\
\hline large & large & 1.385 & $2.883 \mathrm{e}-2$ & $1.007 \mathrm{e}-2$ & $5.560 \mathrm{e}-3$ & $2.841 \mathrm{e}-2$ \\
small & large & 1.385 & $2.889 \mathrm{e}-2$ & $1.011 \mathrm{e}-2$ & $5.541 \mathrm{e}-3$ & $2.845 \mathrm{e}-2$ \\
small & small & 1.385 & $2.889 \mathrm{e}-2$ & $1.010 \mathrm{e}-2$ & $5.545 \mathrm{e}-3$ & $2.845 \mathrm{e}-2$ \\
\multicolumn{2}{c}{ APH } & 1.308 & $2.709 \mathrm{e}-2$ & $1.035 \mathrm{e}-2$ & $4.328 \mathrm{e}-3$ & $2.558 \mathrm{e}-2$ \\
\hline \hline
\end{tabular}

again the largest difference in the $S$-matrix when comparing to the best calculation. The figure shows that the Born errorestimate (the dash-dot line) clearly correlates with the error in the $\boldsymbol{S}$-matrix. The dashed line represents the error in the cumulative reaction probability $\left[N^{0}(E)\right]$. Notice that even though there are 95 open channels in arrangement $1, N^{0}(E)$ is already converged to within about $1 \%$ in a calculation with 25 open DWs and the largest absolute change in any of the $\boldsymbol{S}$-matrix elements (elastic, inelastic, or reactive) is less than $10^{-4}$ for 43 open DWs. The dotted curve shows the singular value corresponding to each open DW. Clearly, the singular value is a good indication of the relative importance of a specific DW.

In arrangement 2 there are only 2 open channels, and we need 2 open DWs. However, Fig. 2(b) shows that for this arrangement it is very important to include at least a few closed DWs: the largest error in the $S$-matrix goes down by three orders of magnitude if three closed DWs are used.

\section{B. Comparison with the APH results}

In Table $\mathrm{V}$ we compare several quantities derived from the $\boldsymbol{P}$-matrix, with the results of PBP. The difference in the cumulative reaction probability $N^{0}(E)$ is about $6 \%$. PBP claim an accuracy of about $1 \%$ for the APH calculation of this quantity. Table $\mathrm{V}$ and the other convergence tests suggest that in the present calculation $N^{0}(E)$ is converged to much better than $1 \%$, possibly to $0.01 \%$. Since the referee suggested that perhaps the fixed grid size is limiting the accuracy of our calculations, we made one additional calculation in which the grid size was extended by $0.25 \mathrm{bohr}$ in each arrangement, to $B_{1}=5.25$ and $B_{2}=B_{3}=5.50 \mathrm{bohr}$. The other parameters were taken as in the "small-small" calculation of Table V, except that in arrangement 1(2) only 40(2) open and 2(4) closed DWs were included. It turns out that this extension of the grid results in a change in $N^{0}(E)$ of less than $10^{-7}$ (compared to the "small-small" calculation). Thus, the "error-bars" of the APH calculation and the present one do not overlap. Still, we think that the agreement may be considered rather good, since the total energy happens to be in the region of a narrow resonance. Therefore, we computed six additional points, scanning through the resonance. The results, together with those of PBP, are shown in Fig. 3. Clearly, the two methods agree both in shape and height of the resonance and the difference, expressed as an energy shift, is at most $0.07 \mathrm{meV}$, which is only a fraction of the width of the resonance. 

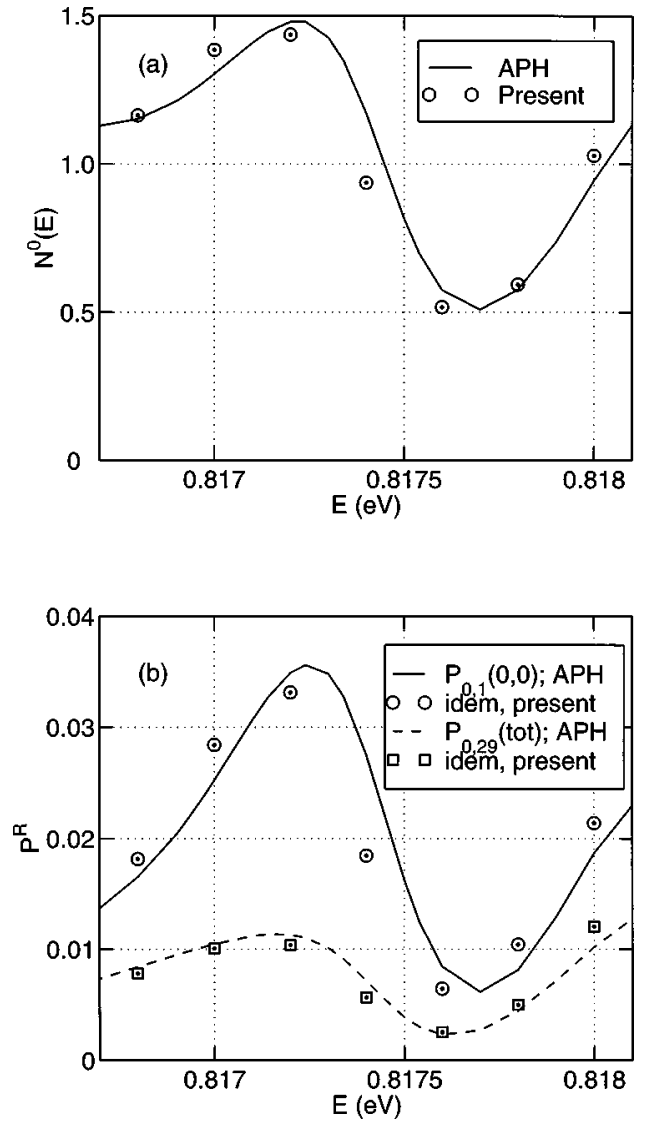

FIG. 3. A comparison between the present calculation (the circles and squares) with the APH calculation of PBP (Ref. 3) (the solid and dashed lines). See also Table V.

\section{Timings}

The main focus in the development of the present method has been the minimization of the size of the interaction region grid under the condition that fully converged results may be obtained by using an optimal set of DWs. Clearly, the present implementation is not yet optimized in all other aspects. For example, it has not been attempted to contract the DVRs ${ }^{13}$ for any of the coordinates. Also, the propagation technique used for the computation of the DWs may be further optimized as mentioned in Appendix B of paper I. Thus, the timings given below are only meant to give an idea of the status of the current implementation.

The most expensive step in the calculation is the iterative solution of the linear equations. On a $250 \mathrm{MHz}$ SUN UltraSparc processor a single iteration takes about $0.4(0.75)$ seconds for the small (large) grid and the number of iterations required for convergence to a residual of $10^{-9}$ is about 32000 (37 000), resulting in 4 (8) cpu-hours for a single right hand side. The total number of right hand sides is equal to the number of closed DWs plus twice the number of open DWs (because of the real and imaginary parts). Note that the work can easily be distributed over several processors (which do not have to be in the same workstation), as long as the number of processor is not larger than the number of right hand sides.

In the current implementation the construction of the DWs takes about 2 (2.5) cpu-days. Again, the work can be distributed over several processors, without appreciable overhead: the propagators for different intervals are computed independently, stored on disk and combined afterwards. This is particularly straightforward, since the coupled channel equations have to be solved for reactive boundary conditions anyhow.

Even though these timings are only preliminary, they suggest that the present method can be expected to be most competitive in a reactive scattering problem where the potential has a deep well and possibly strong long range interactions, but where the total number of open channels is limited. The latter will be the case at a total energy just above the threshold in a reaction which is neither very endoergic nor exoergic (i.e., unlike the $\mathrm{H}+\mathrm{O}_{2}$ reaction, which is endoergic by about $0.71 \mathrm{eV})$.

\section{CONCLUSION}

In paper I a variational method is presented in which the interaction region wave function is represented on a grid. The size of the grid is minimized by using DWs. The expansion of the DWs includes both open and closed channels, allowing the DWs to be fully converged solutions of the Schrödinger equation in the external regions. The number of open DWs is minimized by choosing the boundary conditions with a singular value decomposition technique, which also guarantees numerical stability of the subsequent variational step in the calculation. Closed DWs are used to represent that part of the $\mathscr{b}^{2}$ basis that cannot be represented on the small interaction region grid.

In the present paper the method is applied to the $\mathrm{H}+\mathrm{O}_{2}$ reaction and the most important features of the method are demonstrated numerically:

- Full convergence is obtained with a grid that only extends 5 bohr in the $\mathrm{H}+\mathrm{O}_{2}$ arrangement and $5.25 \mathrm{bohr}$ in the $\mathrm{O}+\mathrm{OH}$ arrangement.

- The number of open DWs needed for full convergence is only about half the number of open channels in this problem.

- Since the grid is relatively small, it is essential to include at least a few closed DWs, in particular in the $\mathrm{O}+\mathrm{OH}$ arrangement.

As an independent check of the method and the implementation the results are compared to those of the APH calculation by Pack et al. Very good agreement is found at a high energy resolution in a region which contains a narrow resonance.

The $S$-matrices computed in the study are available for benchmark purposes from the author. ${ }^{14}$

\section{ACKNOWLEDGMENTS}

The author thanks A. van der Avoird for valuable discussions and for critically reading the manuscript. Russ Pack is gratefully acknowledged for sending the results that are used for comparison in Fig. 3. This work was supported by the Royal Netherlands Academy of Arts and Sciences (KNAW). 
${ }^{1}$ C. Leforestier and W. H. Miller, J. Chem. Phys. 100, 733 (1994).

${ }^{2}$ D. H. Zhang and J. Z. H. Zhang, J. Chem. Phys. 101, 3671 (1994).

${ }^{3}$ R. T Pack, E. A. Butcher, and G. A. Parker, J. Chem. Phys. 102, 5998 (1995); idem, J. Chem. Phys. 99, 9310 (1993).

${ }^{4}$ G. C. Groenenboom, J. Chem. Phys. 108, 5670 (1993), preceding paper.

${ }^{5}$ M. R. Pastrana, L. A. M. Quintales, J. Brandão, and A. J. C. Varandas, J. Phys. Chem. 94, 8073 (1990).

${ }^{6}$ B. Kendrick and R. T Pack, J. Chem. Phys. 106, 3519 (1997).

${ }^{7}$ R. Fei, X. S. Zheng, and G. E. Hall, J. Phys. Chem. A 101, 2541 (1997).

${ }^{8}$ G. C. Groenenboom and D. T. Colbert, J. Chem. Phys. 99, 9681 (1993).
Note the mistake in the signs between the two terms in the first and second part of Eq. (48) (see also Ref. 10).

${ }^{9}$ C. Schwartz, J. Math. Phys. 26, 411 (1985).

${ }^{10}$ D. T. Colbert and W. H. Miller, J. Chem. Phys. 96, 1982 (1992).

${ }^{11}$ G. C. Groenenboom (in preparation).

${ }^{12}$ M. Bramley and T. Carrington, Jr., J. Chem. Phys. 99, 8519 (1993).

${ }^{13}$ D. O. Harris, G. G. Engerholm, and W. D. Gwinn, J. Chem. Phys. 43, 1515 (1965).

${ }^{14}$ The $S$-matrices are available from the author in various electronic formats, e-mail: gerritg@theochem.kun.nl, http://www.theochem.kun.nl/ gerritg. 